\title{
6 Empirische Beispiele für Konflikte um Zugang zu Land im Kontext von land grabbing
}

In diesem Kapitel stelle ich die empirischen Fälle, die als Grundlage meiner Analyse dienen, dar. Diese Fälle sind die Konflikte um die agrarindustriellen Projekte ScanFarm Ltd in der Ashanti Region und BioFuel Africa Ltd in der Northern Region. Zunächst gebe ich einen Überblick über die Entstehung sowie den Verlauf der Projekte bis Anfang 2017, dem Zeitpunkt meines letzten Feldforschungsaufenthalts. Anschließend gehe ich auf drei Aspekte näher ein. Diese drei Aspekte sind der Ablauf der Landvergabe an die Unternehmen, die mit der Landvergabe verbundenen Konflikte sowie die in die Konflikte involvierten Akteure und ihr Handeln in den Konflikten.

\subsection{Das ScanFarm-Projekt - Landvergabe, Konflikte, Konfliktakteure}

2007 initiierten Thor Hesselberg, der Geschäftsführer von ScanFuel Ghana Ltd und drei weitere norwegische Mitbegründer des Unternehmens gemeinsam mit einem ghanaischen Geschäftspartner die Verhandlungen über einen Pachtvertrag für ein agrarindustrielles Jatropha-Projekt in der Gegend um die Dörfer Dukusen, Nsonyameye und Baamaa. Diese befinden sich im Asante Akim North District in der Ashanti Region unweit der Distrikthauptstadt Agogo. ScanFuel Ghana Ltd. ist ein Tochterunternehmen des im selben Jahr gegründeten norwegischen Konzerns ScanFuel AS Norway. Seine Hauptinvestoren sind der norwegische Unternehmer Stale Kyllingstad sowie das norwegische Investmentunternehmen IKM Invest, das Teil der Unternehmensgruppe IKM ist. Ebenso wie die Gründungsmitglieder kommen die Investoren aus der Öl- und Gasindustrie (Roelf 21.11.2008). Mit dem Ziel, die Energiepflanze Jatropha für die Produktion von Agrarkraftstoff anzubauen, 
Abbildung 6: Karte der Regionen Ghanas und den Projektstandorten von ScanFarm und BioFuel Africa

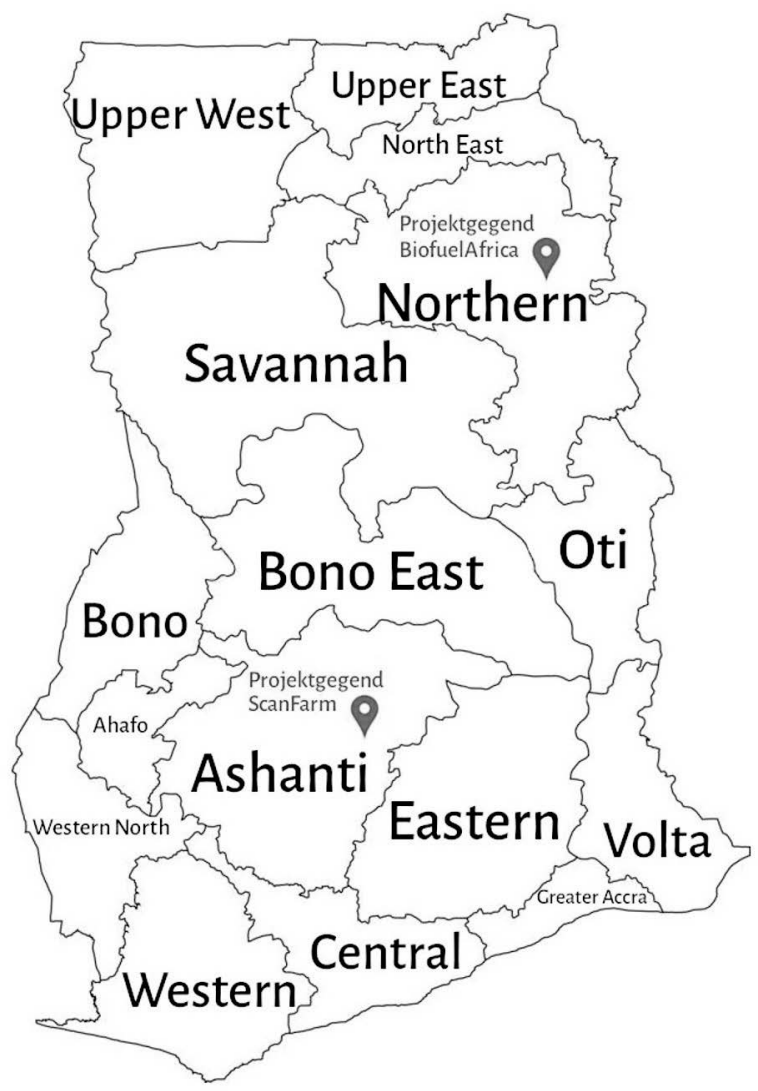

Quelle: Eigene Darstellung

strebte das Unternehmen die Aneignung von etwa 400.000 Hektar Land an. 60 Prozent der Fläche sollten für den Anbau von Jatropha und mindestens 30 Prozent für den Anbau von Nahrungsmitteln genutzt werden. Die verbleibende Fläche sollte als Biodiversitäts-Zone unbewirtschaftet bleiben (Dogbevi 30.08.2010; Roelf 21.11.2008). Im November 2008, auf der Biofuel Markets Africa Conference in Cape Town, Südafrika, berichtete der Geschäftsführer 
des Unternehmens, Thor Hesselberg Vertreter*innen der Presse, ScanFuel habe einen Vertrag über die entsprechende Fläche von 400.000 Hektar abgeschlossen und plane zunächst auf 10.000 Hektar Jatropha anzubauen. Weitere 10.000 Hektar seien für die Produktion von Nahrungsmitteln für den lokalen Markt vorgesehen. Mit der Zeit solle der Anbau ausgeweitet werden. Bis 2015 plane ScanFuel 5000 Barrel Jatropha-Rohöl pro Tag zu produzieren (Roelf 21.11.2008).

Entgegen der Aussage des ScanFuel-Geschäftsführers kam ein Pachtvertrag über die 400.000 Hektar nie zustande. Auch das Vorhaben, Jatropha anzubauen, gab das Unternehmen nach nur einem Jahr auf. Der Rückzug einiger Investoren im Zuge der Finanzkrise, steigende Ölpreise um 2009 und Jatropha-Erträge, die weit hinter dem erwarteten Maß zurückblieben, bewegten das Unternehmen dazu, seine Produktionsstrategie und in diesem Zuge auch seinen Namen zu ändern ${ }^{1}$.

Abbildung 7: Karte der Projektgegend von ScanFarm

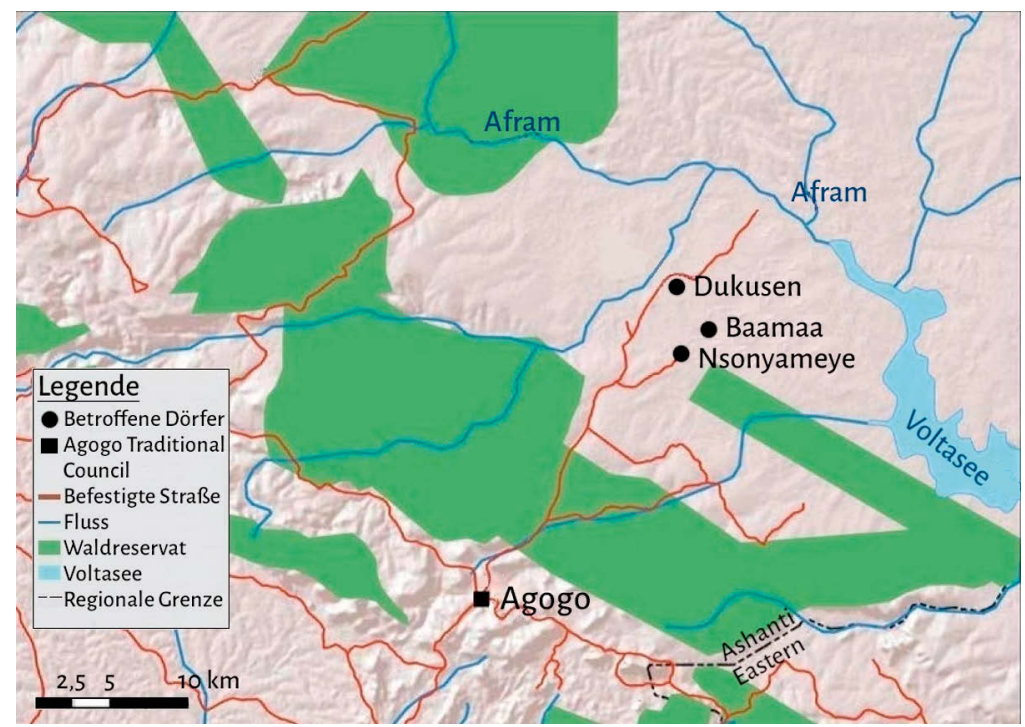

Quelle: Boamah 2014a: 416, eigene Bearbeitung.

1 Ehemaliges Vorstandsmitglied von ScanFarm, Interview am 6.5.2016. 
Seit 2009 ist das Unternehmen unter dem Namen ScanFarm registriert, den ich im weiteren Verlauf des Buches verwende. Seither produziert das Unternehmen vornehmlich Mais ${ }^{2}$. Diesen bezeichnet das ghanaische Landwirtschaftsministerium (Ministry of Food and Agriculture) als priority crop - eine Nutzpflanze, dessen Nachfrage insbesondere als Nahrungsmittel in den letzten Jahren erheblich angestiegen ist und dessen Anbau gefördert werden soll, um eine größere Ernährungssouveränität Ghanas zu ermöglichen ${ }^{3}$. Mais zählt zu den flex crops, ein Terminus der Agrarrohstoffe bezeichnet, die je nach Nachfrage und Marktpreis flexibel als Nahrungsmittel oder für die energetische und industrielle Nutzung weiterverarbeitet werden können (Borras et al. 2016). ScanFarm produziert nach eigenen Angaben ausschließlich für den nationalen Markt, wobei insbesondere Geflügelproduzent"innen und Brauereien die Ernte aufkaufen ${ }^{4}$. 2010, ein Jahr nach der Umstellung auf Mais, erhielt das Unternehmen, das zu diesem Zeitpunkt noch nicht über einen staatlich registrierten Pachtvertrag verfügte, vom ghanaischen Landwirtschaftsministerium die Auszeichnung als National Best Maize Farmer. Seit 2016 ist ScanFarm im Besitz eines staatlich registrierten Pachtvertrags. 2017, zur Zeit meines letzten Feldforschungsaufenthalts, umfasste dieser eine Fläche von 1250 Hektar, wovon das Unternehmen 700 Hektar bewirtschaftete. Ziel des Unternehmens war es, diese Fläche auszuweiten, sobald die gesamten 1250 Hektar für den Anbau genutzt werden ${ }^{5}$.

\section{Landvergabe}

Die Vergabe des Lands in der Gegend von Dukusen, Nsonyameye und Baamaa an ScanFarm geht auf die Initiative von Kyie Bafour zurück, der selbst aus Agogo stammt und bis 2009 Vorstandsmitglied des Unternehmens war. Er hatte die norwegischen Gründungsmitglieder von ScanFarm über riesige Flächen verfügbaren Lands in seiner Heimatgegend informiert und war maßgeblich in die Aneignung des Lands involviert. Zunächst wandte er sich an die paramount chiefs von Gira, Kumawu, Atebubu und Agogo, da das von diesen chiefs verwaltete Land gemeinsame Grenzen hat. Alle vier begrüßten das Vorhaben

\footnotetext{
Geschäftsführer ScanFarm, Interview am 4.4.2016.

Programmleiter Ministry of Food and Agriculture, Interview am 23.3.2016.

Geschäftsführer ScanFarm, Interview am 4.4.2016.

Ceschäftsführer ScanFarm, Interview am 13.3.2017.
} 
und stellten große Flächen dafür in Aussicht ${ }^{6}$. Da sich das Projekt langsam entwickeln sollte, strebte ScanFarm zunächst nur Verhandlungen mit dem Agogo Traditional Council (ATC) an. Dieses Vorhaben wurde maßgeblich dadurch erleichtert, dass Kyie Bafours Onkel der sub-chief von Dukusen - einem der Dörfer in der späteren Projektgegend - war. Dadurch hatte Kyie Bafour direkten Zugang zu traditionellen Institutionen der Landverwaltung. Mit seiner Einschätzung, das Projekt bringe Entwicklung in Form neuer Infrastruktur (wie Straßen, Schulen, Krankenstationen, Wasserdämmen, Brunnen, Elektrizität etc.), sauberer Energie in Zeiten der Energiekrise und des Klimawandels sowie Beschäftigungsmöglichkeiten für die lokale Bevölkerung, konnte er den sub-chief schnell von dem Vorhaben überzeugen ${ }^{7}$. Da dieser nur über sein eigenes stool land frei entscheiden kann und ScanFarm Zugang zu einer Fläche erlangen wollte, die weit über dessen Grenzen hinausging, verwies der subchief von Dukusen das Unternehmen mit seinem Anliegen an den paramount chief. Diesem ist er zu Loyalität verpflichtet, da er derjenige ist der ihn enstooled hat und dem er seine Position zu verdanken hat ${ }^{8}$. Der paramount chief sicherte den Investoren eine Fläche von 19.000 Hektar Land für eine Dauer von 50 Jahren zu. Im Gegenzug soll er laut Aussagen des Geschäftsführers von ScanFarm sowie eines Mitarbeiters des lokalen Landwirtschaftsministeriums eine beträchtliche Summe an drink money erhalten haben ${ }^{9}$. Den genauen Betrag wollten weder der Geschäftsführer des Unternehmens noch die Vertreter des ATC und des CLS nennen. Die zwei ghanaischen Wissenschaftler Elias Danyi Kuusaana und Kaderi Noagah Bukari (2015: 53) sprechen in ihrer Studie zu Konflikten um Land im Asante Akim North District, im Rahmen derer sie auch zu ScanFarm forschten, von 23.000 US-Dollar. 2008 unterzeichneten ScanFarm und der ATC ein Abkommen (memorandum of understanding) ${ }^{10}$. In diesem einigten sich die Parteien auf eine Pachthöhe von einem Dollar pro Hektar und Jahr in 2010, eine jährliche Steigerung von 50 Cent und eine maximale Pachthöhe von 3,50 US-Dollar im Jahr 2014. Darüber hinaus gewährte das Abkommen dem Unternehmen uneingeschränkten Zugang zu jeglichen

$6 \quad$ Ehemaliges Vorstandsmitglied ScanFarm, Interview am 6.5.2016.

7 Sub-chief von Dukusen, Interview am 19.4.2016.

8 Sub-chief von Dukusen, Interview am 19.4.2016.

9 Geschäftsführer ScanFarm, Interview am 4.4.2016; Abteilungsleiter des Regional Office of the Administrator of Stool Lands der Ashanti Region, Interview am 14.4.2016.

10 Geschäftsführer ScanFarm, Interview am 4.4.2016; Registerführer des Agogo Traditional Council und Leiter des Agogo Customary Land Secretariat, Interview am 20.4.2016 
Wasservorkommen auf dem Land und betonte, dass potenzielle Konflikte zunächst durch den ATC bearbeitet werden sollten (Kuusaana/Bukari 2015: 56). Die sub-chiefs und Landnutzer"innen der sich auf dem Land befindenden Dörfer setzte der paramount chief darüber nicht in Kenntnis. Erst nachdem die Investoren und der ATC das Abkommen über die 19.000 Hektar geschlossen hatten, berief der paramount chief die sub-chiefs aus der Gegend zu einem Treffen ein. Im Rahmen dieses Treffens setzte er sie über seine Entscheidung in Kenntnis, sein vermeintlich unbewirtschaftetes stool land - das in offiziellen Dokumenten wie dem Memorandum of Understanding (Absichtserklärung zwischen Unternehmen und Landnutzer*innen) auch als grass land, marginal land oder idle land bezeichnet wird - an ScanFarm zu verpachten. Stool land unterliegt im Gegensatz zu community land der alleinigen Kontrolle durch den Inhaber des jeweiligen stools - in diesem Fall dem paramount chief. Das berechtigt ihn dazu, Verhandlungen über die Vergabe dieses Lands allein zu führen. Die Entscheidung, nur stool land und kein community land zu verpachten, rechtfertigte daher die Entscheidung, die sub-chiefs und Landnutzer*innen zunächst nicht in die Verhandlungen einzubeziehen ${ }^{11}$.

Im Jahr 2008, nachdem ScanFarm und der ATC den Pachtvertrag unterzeichnet hatten, begann das Unternehmen mit der Erlaubnis des paramount chiefs auf 350 Hektar Jatropha anzubauen ${ }^{12}$. Als das Unternehmen 2010 den Anbau von Jatropha beendete, um fortan Mais zu produzieren, wies ein Gutachten darauf hin, dass der Boden in der Gegend sich nicht für das Vorhaben eignete. Daher begann das Unternehmen andere Flächen, die ihm laut Abkommen zustanden, zu erschließen ${ }^{13}$. Dabei stellte sich heraus, dass das durch das Unternehmen genutzte Land nicht wie angekündigt ausschließlich das stool land des paramount chiefs war, sondern auch community land der Dörfer Dukusen, Nsonyameye und Baamaa (Boamah/Overå 2016: 117) umfasste. Der Anbau von Jatropha fand in der Gegend von Dukusen statt. Im Zuge der Umstellung auf Mais begann das Unternehmen auch Grenzen zum community land von Nsonyameye und Baamaa zu überschreiten. Die Landnutzer*innen aus diesen Dörfern realisierten erst nach Beginn der Bearbeitung des Lands durch das Unternehmen, dass ihr Land von dem Projekt betroffen war. Da

\footnotetext{
11 Registerführer des Agogo Traditional Council und Leiter des Agogo Customary Land Secretariat, Interview am 20.4.2016.

12 Geschäftsführer ScanFarm, Interview am 4.4.2016.

13 Geschäftsführer ScanFarm, Interview am 4.4.2016.
} 
es sich bei den Dörfern um farming communities handelt, leben viele der Landnutzer*innen nur in der Anbau- und Erntesaison dauerhaft dort. Die meisten haben ihren permanenten Wohnort in der nahe gelegenen Stadt Agogo. Als sie Kenntnis über die Arbeiten des Unternehmens auf ihrem Land erlangten, waren ihre Felder teilweise schon gepflügt, die Ernte war zerstört, Bäume gefällt und natürliche Grenzmarkierungen entfernt ${ }^{14}$.

Erst nach Beginn der Nutzung des Lands bemühte sich das Unternehmen, den Pachtvertrag bei der Regional Lands Commission registrieren zu lassen und initiierte alle dafür nötigen Schritte wie eine Umwelt- und Sozialverträglichkeitsprüfung (environmental and social impact assessment, ESIA), die Ankündigung des Projekts in den betroffenen Dörfern und ein offizielles Treffen aller Interessengruppen. Aus Unterlagen der Lands Commission geht hervor, dass die 19.000 Hektar nicht nur traditionell verwaltetes Land umfassten. In ihren Untersuchungen stellte die Regional Lands Commission fest, dass ca. 6000 Hektar des Lands bereits auf den ghanaischen Geschäftsmann Bernard Offori registriert waren (Kuusaana/Bukari 2015). In der Konsequenz reduzierte die Lands Commission die Fläche, die für die Registrierung infrage kam, auf 13.000 Hektar (Brief der Lands Commission an ScanFarm, Kumasi, 30.09.2009). In der Zwischenzeit begannen auch einige der Landnutzer"innen und sub-chiefs Einspruch bei dem Unternehmen und der Lands Commission gegen die Vergabe ihres Lands zu erheben. Dabei erhielten sie teilweise Unterstützung durch NGOs wie ActionAid Ghana und das FoodSPAN Netzwerk, die im Rahmen ihrer Advocacy-Arbeit über land grabbing aufklärten und zu Widerstand aufriefen (ActionAid Ghana 2011; Yirrah 2012). Aufgrund des Konflikts verzögerte sich die Registrierung des Pachtvertrags und schließlich lehnte die National Lands Commission, die Pachtverträgen ab einer Größe von 1000 acres $^{15}$ zustimmen muss, sie ab. Als Grund dafür gab die National Lands Commission an, sie erachte die Fläche für das Vorhaben als zu groß. 2016 kam es schließlich doch noch zur Registrierung des Pachtvertrags zwischen ScanFarm und dem ATC. Diese schloss von den ursprünglichen 19.000 Hektar nur 1250 Hektar ein ${ }^{16}$, wobei es sich weiterhin um Land handelt, das zuvor von Kleinbäuerinnen und -bauern genutzt wurde.

Landnutzer*innen aus Nsonyameye, Baama und Dukusen, Interviews am 24., 25., 26. und 27.4.2016. 


\section{Konflikte}

Der Konflikt um das ScanFarm-Projekt entstand, als einige Landnutzer*innen realisierten, dass das Unternehmen ihr Land ohne ihre Zustimmung nutzte.

Um die Angelegenheit zu klären, wandten sich die Landnutzer*innen an die jeweiligen sub-chiefs, die auf Dorfebene für die Schlichtung von Konflikten zuständig sind. Die sub-chiefs, die über die Vergabe des vermeintlichen stool lands an ScanFuel informiert waren, gingen zunächst davon aus, dem Ganzen liege ein Missverständnis zugrunde und das Unternehmen habe versehentlich die Grenzen zu ihrem Land übertreten ${ }^{17}$. Sie berichteten daher dem paramount chief darüber, der beteuerte, er habe kein community land an ScanFarm vergeben. Um dies zu bestätigen schickte der paramount chief ein Mitglied des Agogo Traditional Council (ATC) gemeinsam mit den sub-chiefs auf das Land. Dabei stellte sich heraus, dass sich das Unternehmen tatsächlich zu einem Teil des community land Zugang verschafft hatte. Ob es sich dabei um ein Versehen des Unternehmens handelte oder ob der paramount chief doch mehr als nur das ihr zum freien Verfügung stehende stool land vergeben hatte - was er weiterhin bestritt - blieb zunächst unklar. Deutlich wurde jedoch, dass er keine Ambitionen hatte, das Unternehmen daran zu hindern, weiterhin auf dem Land der Landnutzer*innen zu arbeiten. Entgegen der Bestrebungen der Landnutzer*innen und sub-chiefs, ihr Land zurückzugewinnen, plädierte er für die weitere Nutzung des Lands durch ScanFarm. Er argumentierte, dies sei im Sinne aller, da das Projekt Entwicklung und Arbeit für die Gemeinschaft bringe $^{18}$. Er riet den sub-chiefs, Entschädigungszahlungen mit dem Unternehmen auszuhandeln, statt ihr Land zurückzufordern ${ }^{19}$. Obwohl der paramount chief das Land offiziell vergeben hatte, unterstützte er die sub-chiefs und Landnutzer*innen bei diesem Vorhaben nicht. Die sub-chiefs von Dukusen, Nsonyameye und Baamaa nahmen daraufhin selbst Kontakt zu dem Unternehmen auf, um Entschädigungszahlungen einzufordern ${ }^{20}$. Da ScanFarm im Zuge der Landnutzung Grenzmarkierungen wie Bäume, Sträucher oder Steine zerstört

Sub-chief von Dukusen, Interview am 19.4.2016, sub-chief von Nsonyameye, Interview am 20.04.2016, sub-chief von Baama, Interview am 20.04.2016.

18 Sub-chief von Dukusen, Interview am 19.4.2016.

19 Sub-chief von Dukusen, Interview am 19.4.2016, sub-chief von Nsonyameye, Interview am 20.4.2016.

20 Sub-chief von Nsonyameye, Interview am 20.4.2016; sub-chief von Baama, Interview am 20.4.2016. 
oder entfernt hatte, war es für die Landnutzer*innen schwierig, ihr Land zu lokalisieren ${ }^{21}$. Um Entschädigungszahlungen fordern zu können, müssen Landnutzer*innen jedoch in der Lage sein in der Anwesenheit von Zeug"innen zu zeigen, welches Stück Land sie zuvor genutzt haben. Auch die Ernte war durch die Arbeiten des Unternehmens zerstört worden. Daher konnten die Landnutzer*innen nicht nachweisen, dass sie das Land zuvor bestellt hatten und für den Verlust der Ernte keine Entschädigungszahlungen fordern ${ }^{22}$.

Im Zentrum des Konflikts standen die intransparente Landvergabe sowie der Verlust der Lebensgrundlage. Mit der Intransparenz der Landvergabe sind die mangelnde Information und Konsultierung sowie die fehlenden Partizipationsmöglichkeiten der Landnutzer*innen und sub-chiefs in Bezug auf die Vergabe des Lands gemeint. Zum Verlust der Lebensgrundlage zählen die Zerstörung von Grenzmarkierungen und Ernte sowie unzulängliche Entschädigungszahlungen.

\section{Konfliktakteure}

Die zentralen Akteure in dem Konflikt um Zugang zu dem durch ScanFarm angeeigneten Land können in drei Akteursgruppen unterteilt werden. Ihre Gemeinsamkeit ist, dass sie Ansprüche auf Zugang zu dem umstrittenen Land erheben, wobei sich ihre Interessen diesbezüglich unterscheiden. Diese drei Akteursgruppen sind das Unternehmen ScanFarm, traditionelle Autoritäten und die Landnutzer*innen. Die Unterteilung der Konfliktakteure in diese drei Gruppen dient der Beschreibung des Konflikts. In der Analyse werde ich zeigen, dass traditionelle Autoritäten und Landnutzer"innen in dem Konflikt nicht als kohärente Gruppe handeln, sondern sich durch Heterogenität auszeichnen und unterschiedliche Interessen verfolgen. Zwischen dem Unternehmen, traditionellen Autoritäten und Landnutzer*innen besteht daher eine Vielzahl unterschiedlicher Konflikte und Allianzen.

Die Gruppe der direkt in den Konflikt involvierten traditionellen Autoritäten umfasst den paramount chief, die sub-chiefs der Dörfer Nsonyameye, Dukusen und Baamaa sowie den ehemaligen sub-chief von Dukusen, der den Pos-

Landnutzerin aus Nsonyameye, Interview am 24.4.2016; Landnutzerin aus Nsonyameye, Interview am 26.4.2016; Landnutzerin aus Nsonyameye, Interview am 28.4.2016; Landnutzerin aus Dukusen, Interview am 16.3.2017; Landnutzer aus Nsonyameye und Vorsitzender der Agogoman mma kuo, Interview am 16.3.2017. 
ten des Informanten innehat. Die sub-chiefs sind für die Vergabe von Land an die Landnutzer*innen der Gemeinschaft zuständig. Sie sind zum Teil selbst Landnutzer*innen und vertraut mit den Begebenheiten vor Ort. Der paramount chief ist für die Verwaltung des Lands zuständig, hat aber selbst keinen direkten Bezug dazu. Das bedeutet, dass er nicht in die Vergabe von Land an Landnutzer*innen involviert ist und nicht mit der Nutzung des Lands vor Ort vertraut ist. Für die kommerzielle Nutzung von Land bedarf es jedoch seines Einverständnisses und er erhebt Anspruch auf einen Teil der Einnahmen daraus $^{23}$. Die ghanaische Verfassung gesteht traditionellen Autoritäten die Rolle von Treuhändern zu, jedoch bezeichnet sich der paramount chief selbst als Eigentümer des Landes (owner of the land) und viele Landnutzer*innen erkennen ihn als solchen $a^{24}$. Seine sub-chiefs bezeichnet der paramount chief als Verwalter (caretaker), die nach seinem Willen handeln müssen ${ }^{25}$ Der ehemalige sub-chief von Dukusen ist der so genannte Informant des paramount chiefs. Er informiert den paramount chief über aktuelle Ereignisse in den Dörfern und war dafür zuständig, ScanFarm das Land zu zeigen (sub-chief aus Nsonyameye, Interview am 20.4.2016).

Die Heterogenität innerhalb der Gruppe der Landnutzer*innen drückt sich in Unterschieden hinsichtlich des Zugangs zu, der Rechte an und der Nutzung von Land aus. Rechte an Land unterscheiden sich zwischen indigenen und migrantischen Landnutzer*innen. Die indigenen Landnutzer*innen des von ScanFarm angeeigneten Lands verfügen über family land, über permanente, mündlich durch den sub-chief gewährte Nutzungsrechte (usufruct) oder über permanente, schriftlich durch den paramount chief bestätigte Nutzungsrechte $^{26}$. Migrantische Landnutzer*innen haben temporäre Nutzungsrechte oder Zugang zu Land über sharecropping-Vereinbarungen mit indigenen Landnutzer*innen oder traditionellen Autoritäten ${ }^{27}$. Jenseits bestehender Rechte an Land unterscheiden sich die Landnutzer*innen dadurch, dass einige ihr

23 Registerführer des Agogo Traditional Council und Leiter des Agogo Customary Land Secretariat, Interview am 20.4.2016.

25 Landnutzer aus Dukusen, Interview am 26.3.2016; Mitarbeiter des Asantehenes Land Secretariat, Interview am 10.3.2017.

26 Landnutzerin aus Nsonyameye, Interview am 24.4.2016; Landnutzerin aus Nsonyameye, Interview am 25.4.2016; Landnutzer aus Nsonyameye und Mitglieder der Agogoman mma kuo, Interview am 16.3.2017; Landnutzer aus Nsonyameye und Vorsitzender der Agogoman mma kuo, Interview am 16.3.2017.

27 Landnutzer*innen aus Nsonyameye, Interviews am 24., 25., 27. und 28.4.2016. 
Land vor der Aneignung durch ScanFarm aktiv selbst nutzten, während andere Einkünfte über das Land erzielten, indem sie es an andere Landnutzer*innen verpachteten oder sharecropping-Vereinbarungen eingingen. Auch die Größe des Lands, zu dem die unterschiedlichen Landnutzer*innen vor der Aneignung des Lands durch ScanFarm Zugang hatten, unterscheidet sich. Interviewpartner*innen berichteten von 4 bis 4000 acres, was einem Äquivalent von 1,6 bis 1619 Hektar entspricht ${ }^{28}$. Dieses Land nutzten sie selbst oder ihre Pächter*innen für den Anbau von Bananen, Papaya, Mango, Melone, Mais, Yams, Maniok, Zwiebeln, Tomaten, Erbsen und Erdnüssen. Außerdem befanden sich auf dem Land Shea- und Johannisbrotbäume, die der Herstellung von Sheabutter und Dawadawa, einem in Westafrika weitverbreiteten fermentierten Gewürz, dienen. Je nach Größe des Lands diente es der reinen Subsistenzwirtschaft oder ermöglichte den Verkauf eines Anteils der Erzeugnisse. Außerhalb der Anbau- und Erntesaison gingen viele der Landnutzer*innen in Agogo Tätigkeiten außerhalb der Landwirtschaft nach, unter anderem als Schneider*innen, Schuster*innen, Schreiner oder Kleinhändler*innen ${ }^{29}$.

Die soziale Differenzierung der Landnutzer*innen und traditionellen $\mathrm{Au}$ toritäten spiegelt sich auch in ihren Reaktionen auf den Verlust des Zugangs zu Land wider. Migrantische Landnutzer*innen, die Zugang zu ihrem Land verloren, schimpften zwar auf das Unternehmen, verließen das Land jedoch, ohne Widerstand zu leisten. »Als Fremder hatte ich nicht viel zu sagen « ${ }^{30}$, erklärte ein migrantischer Landnutzer aus dem Norden Ghanas, dessen Familie seit zwei Generationen in der Gegend lebt ${ }^{31}$. Indigene Landnutzer*innen mit permanenten, nicht formalisierten Rechten an Land erkundigten oder beschwerten sich zunächst bei dem jeweiligen sub-chief, der die Beschwerden an den paramount chief weiterleitete. Als sich daraufhin nichts an der Situation änderte, machten sie Gebrauch von konfrontativeren Strategien. Einige weigerten sich ihr Land zu verlassen und drohten mit dem Einsatz von

28 Landnutzer*innen aus Nsonyameye und Dukusen, Interviews am 24., 25, 26., 27. und 28.4. 2016 sowie am 16.3.2017; sub-chief von Nsonyameye, Interview am 20.4.2017.

29 Landnutzer*innen aus Nsonyameye, Interviews am 24., 25., und 27.4.2016; Landnutzerin aus Dukusen, Interview am 16.3.2017; Landnutzer aus Nsonyameye und Vorsitzender der Agogoman mma kuo, Interview am 16.3.2107; sub-chief von Dukusen, Interview am 19.4.2016.

30 Dieses Zitat sowie alle nachfolgenden Zitate aus Interviews sind von mir aus dem Englischen übersetzt.

31 Landnutzer aus Nsonyameye, Interview am 25.4.2016. 
Waffen $^{32}$. Andere nahmen an Demonstrationen teil, um ihr Land zurückzufordern ${ }^{33}$. Im April und Juni 2010 demonstrierten zahlreiche Landnutzer*innen und Bewohner*innen von Agogo gegen land grabbing und verschafften sich Zugang zum Palast des paramount chiefs (Boamah 2015; Kuusaana 2016). Schließlich forderten zahlreiche Landnutzer*innen gemeinsam mit den subchiefs von Nsonyameye und Baamaa Entschädigungszahlungen von ScanFarm für den Verlust ihres Lands und die Zerstörung der Ernte. Diese Gruppe agierte jedoch nicht als geschlossene Einheit. Einige akzeptieren das erste Angebot des Unternehmens und nahmen darüber hinaus Tätigkeiten an, die das Unternehmen ihnen anbot. Andere, zu denen auch die sub-chiefs gehörten, gaben sich nicht damit zufrieden und forderten höhere Entschädigungszahlungen $^{34}$. Wieder andere gaben die Verhandlung mit ScanFarm auf, nachdem sie feststellten, wie wenig Entschädigung das Unternehmen ihnen für das Land zahlen wollte. Dies verdeutlich auch die Aussage eines indigenen Landnutzers aus Nsonyameye: "Als mir bewusst wurde, dass die Entschädigungszahlungen gering waren, beschloss ich, meine Zeit nicht zu verschwenden. Seitdem bin ich nie wieder zum Unternehmen gegangen, da ich es für nutzlos halte « ${ }^{35}$. Landnutzer*innen mit formalisierten, also schriftlich durch den paramount chief anerkannten, Landrechten konfrontierten zunächst den paramount chief mit dem Verlust ihres Lands an ScanFarm. Der paramount chief bestätigte diese Rechte, unternahm jedoch nichts, um das Unternehmen an der Nutzung des Lands zu hindern. Nachdem das Unternehmen den Forderungen der Landnutzer*innen, ihnen ihr Land zurückzugeben, nicht nachkam, erhoben einige von ihnen 2014 über das staatliche Rechtssystem Klage gegen das Unternehmen ${ }^{36}$. Einige von ihnen waren Mitglieder des zivilgesellschaftlichen Netzwerks Agogoman mma kuo (weltweite Bürger*innen von Agogo), die sich für gesellschaftspolitische Belange der Bürger*innen von Agogo einsetzt. Die Agogoman mma kuo engagierten sich ebenfalls für Entschädigungszahlungen,

32 Landnutzer aus Nsonyameye und Vorsitzender der Agogoman mma kuo, Interview am 16.3.2017; Geschäftsführer ScanFarm, Interview am 4.42016.

33 Landnutzer aus Nsonyameye, Interview am 28.4.2016; Landnutzer aus Nsonyameye und Vorsitzender der Agogoman mma kuo, Interview am 16.3.2017.

34 Sub-chief von Nsonyameye, Interview am 20.4.2016; sub-chief von Baamaa, Interview am 20.4.2016.

35 Landnutzer aus Nsonyameye, Interview am 28.4.2016.

36 Landnutzer aus Nsonyameye und Mitglieder der Agogoman mma kuo, Interview am 16.3.2017. 
mehr Arbeitsplätze für Menschen aus der Gegend und eine größere Transparenz der Landvergabe, beispielsweise durch Einsicht in das Abkommen zwischen dem paramount chief und dem Unternehmen ${ }^{37}$. Darüber hinaus unterbreiteten sie dem Asantehene, dem traditionellen Oberhaupt der Ashanti, eine Petition zur Amtsenthebung (destoolment) des paramount chiefs von Agogo Nana Akuo Sarpong. Als Grund für die Petition führten sie an, er sei »kläglich an der Aufgabe gescheitert, das Land, für das die Vorfahren gekämpft und welches sie hinterlassen haben zu schützen sowie die Bürgerinnen und Bürger jederzeit $\mathrm{zu}$ beschützen und zu verteidigen $^{38}$. Dieses Vorhaben blieb jedoch erfolglos.

\subsection{Das BioFuel Africa-Projekt - Landvergabe, Konflikte und Konfliktakteure}

BioFuel Africa Ltd ist eine Tochtergesellschaft des norwegischen Unternehmens BioFuel Norway und seit 2007 in Ghana registriert (Bruce 2008). Ziel der Gründer von BioFuel Africa war es, in der Northern Region Ghanas die weltweit größte Jatropha-Plantage aufzubauen (Nyari 2008). Die Hauptinvestoren waren das finnische Staatsunternehmen Neste Oil und der norwegische Gasund Ölproduzent STATOIL (seit 2018 Equinor) (Nygaard/Bolwig 2018). Um geeignetes Land dafür zu finden, begannen sie 2005 in ghanaischen Zeitungen $\mathrm{zu}$ inserieren. Auf diese Weise entstand der Kontakt zu ihrem späteren ghanaischen Geschäftspartner, Herrn Awal. Dieser unterstützte die norwegischen Gründer von BioFuel Africa dabei, Land im damaligen Yendi District (heute Mion District) und im Central Gonja District der Northern Region für den Anbau von Jatropha zu identifizieren und den Kontakt zu den traditionellen Autoritäten, die das Land verwalteten, herzustellen. Vorteilhaft dafür war, dass er der Enkelsohn des Tijo Naa (chief von Tijo) ist, der später für die Vergabe des Lands an BioFuel Africa verantwortlich war. Seit 2007 ist BioFuel Africa als Unternehmen in Ghana registriert und erlangte laut unterschiedlichen Quellen sowie Aussagen des Geschäftsführers von BioFuel Africa (Kol-

\footnotetext{
37 Landnutzer aus Nsonyameye und Vorsitzender der Agogoman mma kuo, Interview am 16.3.2017.

38 Landnutzer aus Nsonyameye und Mitglieder Agogoman mma kuo, Interview am 16.3.2017; Landnutzer aus Nsonyameye und Vorsitzender der Agogoman mma kuo, Interview am 16.3.2017; Auszug aus der Petition, Übersetzung durch die Autorin)
} 
nes 2009) Zugang zu insgesamt 23.760 Hektar Land in den beiden Distrikten. 2008 erhielt es nach der Durchführung einer Umwelt- und Sozialverträglichkeitsprüfung für die gesamte Fläche eine Genehmigung (environmental permit) durch die ghanaische Umweltbehörde (Environmental Protection Agency, EPA) (Boamah 2011b). Seit 2009 sind 10.696 Hektar im Mion District bei der Lands Commission registriert (Kolnes 2009). Das Vorhaben im Central Gonja District hingegen gab BioFuel Africa aufgrund von Widerstand seitens der Bevölkerung schnell auf und konzentrierte sich ausschließlich auf das Land im Mion District (Tsikata/Yaro 2011). Das Unternehmen gibt an, insgesamt einen Pachtvertrag über 154.778 Hektar abgeschlossen zu haben (Kolnes 2009; SGS Environment 2009). In einigen Presseberichten und Publikationen von NGOs ist demgegenüber von einer Flächengröße von 38.000 Hektar die Rede (Knaup 05.09.2008; Nyari 2008). Unklar bleibt, woher diese Daten stammen. Zusätzlich zu dem Land in der Northern Region verfügt das Unternehmen über 840 Hektar Land in der Volta Region nahe Sogakope. Diese Fläche nutzte es als Testfarm für den Jatropha-Anbau (SGS Environment 2009).

Abbildung 8: Karte der betroffenen Dörfer des BioFuel Africa-Projekts

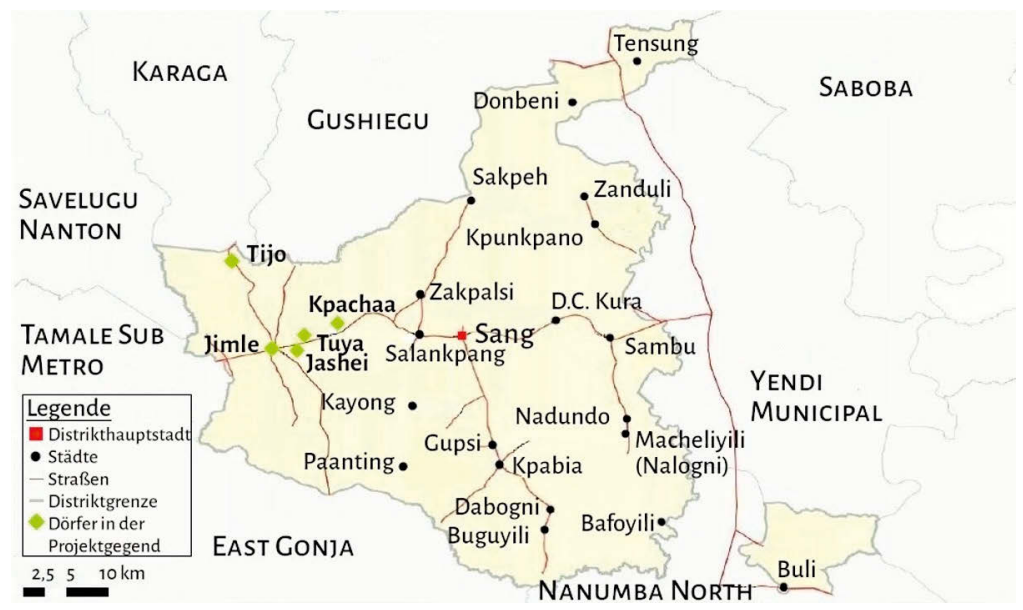

Quelle: Ghana Statistial Service 2014: 2. Eigene Darstellung

Im Jahr 2007 begann BioFuel Africa in der Gegend um die Dörfer Kpachaa, Jashei, Jimle und Tuya auf einer Fläche von 480 Hektar mit dem JatrophaAnbau (SGS Environment 2009). Während der Hochsaison beschäftigte das 
Unternehmen 320 Mitarbeiter*innen, von denen zwei Drittel aus Tamale und ein Drittel aus den Dörfern in der Projektgegend kamen (Bruce 2008). BioFuel Africa strebte an, diese Fläche bis 2011 auf 17.000 Hektar auszuweiten (Kolnes 2009). Dazu kam es jedoch nicht. Vielmehr sorgte das Projekt 2008 nationale und international für negative Schlagzeilen. Grund dafür war die Entscheidung des Unternehmens, ohne Genehmigung durch die Lands Commission und die Umweltbehörde sowie ohne Konsultierung der Landnutzer*innen zu beginnen, Felder für die agrarindustrielle Bewirtschaftung vorzubereiten. Dabei wurden zahlreiche Bäume gefällt, die für die Landnutzer*innen von hohem wirtschaftlichem Wert waren. Dies nahmen insbesondere die NGO Action Aid Ghana und die zivilgesellschaftliche Organisation Regional Advisory and Information Network Systems (RAINS) zum Anlass, um gegen das Projekt zu mobilisieren und zu breitem Widerstand gegen land grabbing aufzurufen (Nyari 2008; Yirrah 2012).

Dennoch produzierte das Unternehmen Ende 2008 das erste noch unkommerzielle Jatropha-Rohöl, ungereinigtes Pflanzenöl, wie es nach der Ölpressung vorliegt (Kolnes 2009). Aufgrund der negativen Presse, bestehender Korruptionsvorwürfe sowie steigender Ölpreise entzog der Gas- und ÖlGigant STATOIL kurze Zeit später, im Jahr 2009, dem Projekt die zugesicherten finanziellen Mittel (Tsikata/Yaro 2011: 21). Aufgrund der fehlenden Finanzierung musste das Unternehmen im März 2009 Konkurs anmelden (persönliche Korrespondenz, Geschäftsführer BioFuel Africa, 15.02.2017). BioFuel Africa baute somit nur zwei Jahre lang Jatropha auf dem Land $a^{39} .2009$ kauften zwei der ehemaligen Gründungsmitglieder, Steinar Kolnes und Arne Helvig, BioFuel Africa auf, mit dem Ziel, fortan Nahrungsmittel zu produzieren. 2010 benannten sie das Unternehmen in Solar Harvest um (Boamah 2011a; Tsikata/Yaro 2011). Seit 2014 erhält Solar Harvest finanzielle Förderung durch das Ghana Commercial Agriculture Project (GCAP) des ghanaischen Landwirtschaftsministeriums. Mithilfe der Förderung will das Unternehmen in Botanga (Kunbungu District, Northern Region) auf 200 Hektar Land ein Bewässerungssystem aufbauen, das den Anbau von Reis, Mais und Soja im Rahmen von Vertragslandwirtschaft ermöglichen soll (SAL 2017). GCAP ist ein durch USAID und die Weltbank finanziertes Projekt zur Förderung einer kommerziellen Landwirtschaft. 


\section{Landvergabe}

Für die Vergabe des Lands an BioFuel Africa spielten insbesondere der ghanaische Geschäftspartner von BioFuel Africa, Adam Awal, der Tijo Naa und sein Anwalt sowie der Regent of Dagbon (in Vertretung des $\mathrm{Ya} \mathrm{Naa}$ ) eine zentrale Rolle. Auf der Suche nach einer geeigneten Fläche für den Anbau von Jatropha wandte sich der ghanaische Geschäftspartner von BioFuel Africa gemeinsam mit den norwegischen Investoren zunächst an den sub-chief von Jimle, seinem Heimatort und überzeugte ihn von dem Vorhaben ${ }^{40}$. Dieser verwies ihn an den Tijo Naa - der vom Status her mit einem paramount chief zu vergleichen ist. Einige elders aus Jimle begleiteten Adam Awal zum Tijo Naa, der bestätigte, dass es sich um seinen Enkelsohn handelte, was für die Gemeinschaft nur Gutes bedeuten könne, und befürwortete die Vergabe von Land (Tsikata/Yaro 2011).

BioFuel Africa initiierte daraufhin eine Veranstaltung im Tamale Cultural Center mit allen zuständigen chiefs, einigen der betroffenen Landnutzer* innen, Angestellten der District Assembly und einigen NGOs, um über das Projekt zu informieren. Sie stellten Busse zur Verfügung, die die Teilnehmenden aus den unterschiedlichen Dörfern in die ca. $50 \mathrm{~km}$ entfernte Regionalhauptstadt Tamale brachten. Im Rahmen dieser Veranstaltung machte der Tijo Naa deutlich, dass er sich von dem Projekt in erster Linie erhoffe, dass es der Gemeinschaft zugutekomme ${ }^{41}$. Im Anschluss an das Treffen besichtigte BioFuel Africa das zur Verfügung stehende Land und engagierte das ConsultingUnternehmen SGS Ghana, um eine Umwelt- und Sozialverträglichkeitsprüfung (ESIA) durchzuführen (SGS Environment 2009). Darüber hinaus organisierte das Unternehmen zwei weitere öffentliche Anhörungen in Tijo und Yendi, wo der Regent of Dagbon seinen Sitz hat. Dieser stimmte der Vergabe des Lands durch den Tijo Naa zu, woraufhin der Tijo Naa, der selbst Analphabet ist, einen Anwalt beauftragte, um den Pachtvertrag mit dem Unternehmen aufzusetzen. Der Pachtvertrag umfasste eine Fläche von 13.800 Hektar, von denen später 10.696 Hektar bei der Lands Commission registriert wurden. Er war auf 25 Jahre begrenzt, jedoch mit Aussicht auf eine Verlängerung von weiteren 25 Jahren nach Ablauf der Frist (Tsikata/Yaro 2011). Die Pacht betrug zwei Ghanaische Cedi (GHS) pro Hektar, was zum damaligen Zeitpunkt

40 Landnutzer Gimle und Gumani, Gruppendiskussion am 10.5.2016.

41 Paramount-chief und elders von Tijo, Gruppendiskussion am 8.5.2016. 
ungefähr einem Wert von knapp einem Euro bzw. etwas mehr als einem USDollar entsprach. Die Pachthöhe ist damit ähnlich wie auch in anderen Fällen großflächiger Landvergabe an internationale Unternehmen. Das zeigt der Vergleich mit anderen Studien, die Projekte in diesem Zeitraum untersucht haben (Kuusaana/Bukari 2015: 56). Die erste Zahlung war für die ersten zwei Jahre angesetzt. Darauf sollten jährliche Zahlungen folgen ${ }^{42}$. 40 Prozent der Pacht gingen an den Regent of Dagbon in Vertretung des Ya Naa. Darüber hinaus gab der Tijo Naa den anderen paramount chiefs aus der Gegend sowie seinen eigenen sub-chiefs zwischen 500 und 1000 GHS, was zum damaligen Zeitpunkt etwa 290 bis 480 Euro entsprach. Die finanzielle Teilhabe der sub-chiefs war unabhängig davon, wessen Land der Pachtvertrag umfasste (Tsikata/Yaro 2011).

Den in der Gegend lebenden Landnutzer*innen, deren Land durch das Projekt betroffen war, bot das Unternehmen drei Optionen an. Sie hatten die Möglichkeit ein anderes Stück Land auszuwählen, das Arbeiter*innen des Unternehmens maschinell für sie roden und pflügen würden oder als Arbeiter*innen für BioFuel Africa zu arbeiten, um ihren Lebensunterhalt zu verdienen. Wenn keine dieser beiden Optionen für sie infrage käme, würde das Unternehmen sie in bar für die Nutzung des Landes bezahlen ${ }^{43}$ (Tsikata/Yaro 2011: 19).

\section{Konflikte}

Anders als im Fall von ScanFarm versuchte BioFuel Africa mit Beginn der Verhandlungen gleichermaßen chiefs und betroffene Landnutzer*innen in den Prozess der Landvergabe einzubeziehen, um Konflikte zu vermeiden. Im Rahmen mehrerer öffentlicher Anhörungen erklärte BioFuel Africa ihr Vorhaben gegenüber Repräsentant"innen der betroffenen Dörfer. Um den Nutzen des Projekts für die Gemeinschaft deutlich zu machen, stellte das Unternehmen Arbeitsplätze und die Verbesserung der Infrastruktur in der Gegend in Aussicht ${ }^{44}$. Während des Höhepunkts der Aktivitäten beschäftigte BioFuel Afri$c a$ insgesamt 116 Arbeiter*innen (67 Männer und 39 Frauen) aus der Projektgegend. Der Großteil der qualifizierten Arbeiter*innen (insgesamt ungefähr

\footnotetext{
42 Paramount-chief und elders von Tijo, Gruppendiskussion am 8.5.2016.

43 Landnutzer*innen und sub-chief von Tuya, Gruppendiskussion am 14.5.2016.

44 Paramount-chief und elders von Tijo, Gruppendiskussion am 8.5.2016.
} 
280) kam jedoch aus Tamale (Bruce 2008: 3). Darüber hinaus errichtete BioFuel Africa eine Mühle in der Gegend von Kpachaa, zwei Dämme in der Nähe von Jashei und Jimle und ebnete die Zubringerstraße nach Jimle. Während der Regenzeit pflügten Arbeiter"innen des Unternehmens für einige Landnutzer*innen Land in Jashei ${ }^{45}$ (ebd.: 4). Trotz der Bemühungen um einen transparenten Ablauf der Landvergabe waren nicht alle Landnutzer*innen und sub-chiefs gleichermaßen informiert ${ }^{46}$ (Ahmed et al. 2018: 576). Auch hatte das Projekt nicht für alle die gleichen positiven und negativen Auswirkungen $^{47}$.

Der Konflikt, der sich im Laufe der Implementierung des Projekts herausbildete, betrifft die Intransparenz in der Kommunikation zwischen den einzelnen chiefs sowie zwischen dem Unternehmen und den Bewohner*innen der einzelnen Dörfer, die Rodung wirtschaftlich genutzter Bäume und die Verschlechterung der Lebensgrundlage der Landnutzer*innen, enttäuschte Erwartungen sowie die Veränderung des Lebensraums.

In Jimle, Jashei, Tuya und Changolinaaya waren die Landnutzer*innen und auch der sub-chief schlecht über das Vorgehen von BioFuel Africa bezüglich der Landnutzung informiert ${ }^{48}$. Außer in Jashei und Changolinaayawaren die Landnutzer*innen in den einzelnen Dörfern zwar über das Projekt in Kenntnis gesetzt worden, wurden im weiteren Verlauf jedoch nicht mehr regelmäßig informiert ${ }^{49}$. Sie erhielten keine Informationen darüber, wann das Projekt starten sollte und auch vom Ende des Jatropha-Anbaus erfuhren sie erst, als die Entscheidung bereits getroffen war. Der Mangel an Informationen ging teilweise auf die unzulängliche Kommunikation zwischen den un-

45 Landnutzer*innen aus Jimle und Gumani, Gruppendiskussion am 10.5.2016; Landnutzer*innen und settler-chief aus Kpachaa, Gruppendiskussion am 11.5.2016; Landnutzer*innen und sub-chief aus Jashei, Gruppendiskussion am 15.5.2016.

46 Landnutzer aus Changolinaaya, Interview am 12.5.2016; Landnutzer und settler-chief aus Parachanayilli, Gruppendiskussion am 13.5.2016; Landnutzer*innen und sub-chief aus Tuya, Gruppendiskussion am 14.5.2016; Landnutzer*innen und sub-chief Jashei, Gruppendiskussion am 15.5.2016.

47 Landnutzer*innen und sub-chief aus Jashei, Gruppendiskussion am 15.5.2016.

48 Landnutzer*innen aus Jimle und Cumani, Gruppendiskussion am 10.5.2016; Landnutzer*innen und sub-chief aus Jashei, Gruppendiskussion am 15.5.201.; Landnutzer*innen und sub-chief aus Tuya, Gruppendiskussion am 14.5.2016; Landnutzer aus Changolinaaya, Interview am 12.5.2016.

49 Landnutzer*innen und sub-chief aus Jashei, Gruppendiskussion am 15.5.2016; Landnutzer aus Changolinaaya, Interview am 12.5.2016. 
terschiedlichen chiefs und teilweise auf fehlende Benachrichtigung durch das Unternehmen zurück ${ }^{50}$ (Bruce 2008).

Im Umfeld der Dörfer Tuya, Jashei, Kpachaa und Parachanayilli rodete BioFuel Africa hunderte Bäume, um die Felder für den Jatropha-Anbau anzulegen ${ }^{51}$. Dabei handelte es sich unter anderem um Sheanuss-Bäume und Johannisbrotbäume, deren Früchte Frauen zu Sheabutter und Dawadawa weiterverarbeiteten. Über den Verkauf der Produkte erwirtschafteten sie zusätzliches Einkommen für die Familie. Die Rodung der Bäume schränkte diese Möglichkeit ein. Die Lebensgrundlage der betroffenen Landnutzer*innen verschlechterte sich zusätzlich, da zuvor anwesende Landwirt*innen aus Tamale aufgrund des BioFuel Africa-Projekts Zugang zu dem von ihnen genutzten Land verloren und die Gegend verlassen mussten. Sie hatten es den Anwohner*innen gestattet, die nach der Ernte auf den Feldern verbleibenden Reste zu sammeln, was für diese eine Möglichkeit darstellte, die eigene Ernte oder knappe Essensvorräte aufzustocken ${ }^{52}$.

Im Zentrum des Konflikts stand darüber hinaus die Veränderung des Lebensraums, die mit der Umsetzung des BioFuel Africa-Projekts einherging. Anwohner*innen der Dörfer berichteten, dass durch die Rodung zahlreicher Bäume starker Wind größere Schäden an den Häusern anrichtete als zuvor ${ }^{53}$ und heftige Regenfälle sowie Dürre stärkere Auswirkungen hätten ${ }^{54}$. Zudem wiesen einige Anwohner*innen darauf hin, dass die Umgebung aufgrund der buschigen Jatropha-Pflanzen - die auch über das Projektende hinweg auf dem Land verblieben, da BioFuel Africa sie nicht entfernte - insbesondere für Kinder gefährlich sei, da sich dort viele Schlangen einnisteten ${ }^{55}$.

50 Landnutzer*innen und sub-chief aus Tuya, Gruppendiskussion am 14.5.2016; Landnutzer*innen und sub-chief aus Jashei, Gruppendiskussion am 15.5.2016.

51 Landnutzer*innen und settler-chief aus Kpachaa, Gruppendiskussion am 11.5.2016; Landnutzer und settler-chief aus Parachanayilli, Gruppendiskussion am 13.5.2016; Landnutzer*innen und sub-chief aus Tuya, Gruppendiskussion am 14.5.2016; Landnutzer*innen und sub-chief aus Jashei, Gruppendiskussion am 15.5.2016.

52 Landnutzer*innen und sub-chief aus Tuya, Gruppendiskussion am 14.5.2016.

53 Landnutzer*innen und settler-chief aus Kpachaa, Gruppendiskussion am 11.5.2016; Landnutzer"innen und sub-chief aus Tuya, Gruppendiskussion am 14.5.2016.

54 Landnutzer und settler-chief aus Parachanayilli, Gruppendiskussion am 13.5.2016.

55 Landnutzer ${ }^{*}$ innen und settler-chief aus Kpachaa, Gruppendiskussion am 11.5.2016. 
Schließlich sind die enttäuschten Erwartungen, die viele Landnutzer*innen an das Projekt hatten relevant für den Konflikt ${ }^{56}$. Auch wenn Landnutzer*innen aus allen betroffenen Dörfern positive Auswirkungen des Projekts wie den verbesserten Zugang zu Wasser oder Arbeitsmöglichkeiten beschreiben, kritisieren viele, dass das Projekt nicht allen Landnutzer*innen gleichermaßen zugutegekommen sei. Nur wenige Personen aus den Dörfern seien eingestellt worden. Insbesondere Frauen, die als Arbeiter*innen für das Projekt unterrepräsentiert waren und ältere Männer, die keine Söhne hatten, die für das Unternehmen arbeiteten, gaben an, sich benachteiligt zu fühlen (Bruce 2008). Weit verbreitet war in den Dörfern außerdem die Meinung, nicht ausreichend an dem Geld, welches das Unternehmen für das Land gezahlte hatte, teilzuhaben ${ }^{57}$.

\section{Konfliktakteure}

Die zentralen Akteure in dem Konflikt um Zugang zu dem durch BioFuel Africa anageeigneten Land können, wie auch im Fall von ScanFarm, in drei Akteursgruppen unterteilt werden. Diese drei Akteursgruppen sind das Unternehmen BioFuel Africa, traditionelle Autoritäten und die Landnutzer*innen. Wie auch im Konflikt um ScanFarm sind traditionelle Autoritäten und Landnutzer*innen keine homogenen Gruppen, sondern sozial differenziert. Unterschiede innerhalb der Gruppen bestehen insbesondere in Bezug auf den Zugang zu Land.

Die Gruppe der traditionellen Autoritäten besteht aus dem Tijo Naa, den sub-chiefs und den settler-chiefs. Der Tijo Naa verwaltet das Land in der Projektgegend. Unter ihm stehen die sub-chiefs, an die der Tijo Naa die Zuständigkeit für die Verwaltung von Teilen seines Lands überträgt. Darüber hinaus gibt es in der Gegend einige settler chiefs, die so genannten settler communities vorstehen. In settler communities leben migrantische Landnutzer*innen, das heißt Landnutzer*innen, die ursprünglich aus einer anderen Gegend kommen. Bei ihrer Ankunft in der Gegend baten sie den zuständigen chief um Land. In der

56 Paramount-chief und elders von Tijo, Gruppendiskussion am 8.5.2016; Landnutzer und settler-chief aus Parachanayilli, Gruppendiskussion am 13.5.2016; Landnutzer*innen und sub-chief von Tuya, Gruppendiskussion am 14.5.2016.

57 Landnutzer*innen aus Nsonyameye, Baama \& Dukusen, Gruppendiskussion am 27.4.2016; Landnutzer*innen und sub-chief von Jashei, Gruppendiskussion am 15.5.2016.6. 
Gegend von BioFuel Africa sind die settler communities Kpachaa, Parachanayilli und Changolinaaya ${ }^{58}$. Der chief von Kpachaa hat das Land für seine Gemeinschaft über den Tijo Naa erhalten, diejenigen in Parachanayilli und Changolinaaya über den Jashei-Naa. Settler chiefs vergeben das Land an die Landnutzer*innen in ihrer Gemeinschaft, haben aber keine permanenten Rechte an Land und müssen sich den Entscheidungen der über ihnen stehenden chiefs beugen $^{59}$.

Die Gruppe der Landnutzer*innen in der Gegend des BioFuel AfricaProjekts besteht aus dort ansässigen Kleinbäuerinnen und -bauern sowie aus Landwirt*innen, die das Land zu kommerziellen Zwecken nutzen, jedoch nicht dort leben. Die Kleinbäuerinnen und -bauern aus Jimle, Jashei und Tuya stammen ursprünglich aus der Gegend, bezeichnen sich selbst als indigen und erheben permanente Ansprüche auf ihr Land ${ }^{60}$. Diejenigen aus Kpachaa, Parachanayilli und Changolinaaya stammen ursprünglich aus anderen Gegenden und akzeptieren, dass ihre Rechte an Land temporär sind und sie keinerlei Entscheidungsbefugnis hinsichtlich der Nutzung des Lands haben ${ }^{61}$. Die Gegend des BioFuel Africa-Projekts ist extrem strukturschwach, mit weit auseinanderliegenden, durch unbefestigte Straßen verbundenen Dörfern von meist wenigen Hundert Einwohner*innen. Die Haupteinkommensquelle der Menschen aus der Gegend des BioFuel Africa-Projekts ist die kleinbäuerliche Landwirtschaft. Vorwiegend Männer bauen Mais, Hirse, Bohnen, Maniok, Erdnüsse, Sojabohnen, Okra und Yamswurzeln für den Eigenbedarf und teilweise auch für den Verkauf $a^{62}$. Da die Anbausaison aufgrund der geringen Regenmengen und der kurz andauernden Regenfälle

58 Landnutzer aus Changolinaaya, Interview am 12.5.2016; Landnutzer*innen und settlerchief aus Kpachaa, Gruppendiskussion am 11.5.2016; Landnutzer und settler-chief aus Parachanayilli, Gruppendiskussion am 13.5.2016

59 Landnutzer"innen und settler-chief aus Kpachaa, Cruppendiskussion am 11.5.2016; Landnutzer und settler-chief aus Parachanayilli, Gruppendiskussion am 13.5.2016.

60 Landnutzer ${ }^{*}$ innen aus Jimle und Gumani, Gruppendiskussion am 10.5.2016; Landnutzer"innen und sub-chief von Tuya, Gruppendiskussion am 14.5.2016; Landnutzer“innen und sub-chief von Jashei, Gruppendiskussion am 15.5.2016.

61 Landnutzer aus Changolinaaya, Interview am 12.5.2016; Landnutzer"innen und settlerchief aus Kpachaa, Gruppendiskussion am 11.5.2016; Landnutzer und settler-chief aus Parachanayilli, Gruppendiskussion am 13.5.2016.

62 Paramount-chief und elders von Tijo, Gruppendiskussion am 8.5.2016; Landnutzer*innen aus Nsonyameye, Baama \& Dukusen, Cruppendiskussion am 27.4.2016; Landnutzer*innen aus Jimle und Cumani, Gruppendiskussion am 10.5.2016; Landnutzer*innen und sub-chief von Tuya, Gruppendiskussion am 14.5.2016. 
zeitlich begrenzt ist, sind die Landnutzer*innen neben der Landwirtschaft auf andere wirtschaftliche Tätigkeiten angewiesen. Außerhalb der Anbauund Erntezeit produzieren sie Holzkohle und betreiben Viehzucht. Die Frauen stellen hauptsächlich Sheabutter und Dawadawa her ${ }^{63}$. Mit dem Verkauf von Tieren, Holzkohle, Sheabutter und Dawadawa überbrücken sie maßgeblich den so genannten hunger gap, die Zeit zwischen dem Aufbrauchen der eigenen Erzeugnisse und dem Beginn der nächsten Ernte (Bruce 2008: 11). Landwirt*innen, die vor Beginn des Projekts dem kommerziellen Anbau von Nutzpflanzen nachgingen, haben ihr Land über den Tijo Naa erhalten. Für die Nutzung des Lands mussten sie einen Teil ihrer Ernte an den chief zahlen. Ebenso wie migrantische Landnutzer*innen haben diese Landwirt*innen keine permanenten Rechte an Land.

Die Reaktionen der Konfliktakteure auf die Aneignung des Lands durch BioFuel Africa unterscheiden sich insbesondere zwischen den in der Gegend lebenden Landnutzer*innen und sub-chiefs sowie den Landwirt*innen, die dort kommerzielle Landwirtschaft betrieben, jedoch aus Tamale kamen. Letzteren kann nach traditionellem Landrecht der Zugang zu dem Land jederzeit und ohne Ersatz verwehrt werden, wenn das Land für andere Zwecke, die dem Wohl der Gemeinschaft dienen, benötigt wird. Das machte der Tijo Naa gegenüber dem Unternehmen deutlich und warnte davor, Entschädigungszahlungen an diese Landnutzer*innen zu leisten (Tsikata/Yaro 2011: 21). Einige von ihnen verließen die Gegend und ließen sich an einem anderen Ort nieder, um dort weiterhin Landwirtschaft zu betreiben. Andere gaben ihre Tätigkeit in der Landwirtschaft auf. Ein Teil von ihnen mobilisierte über Zeitungsartikel (Nyari 2008; The New Humanitarian 2009) und Radiosendungen gegen das Unternehmen und erlangte die Aufmerksamkeit nationaler sowie internationaler NGOs, welche die Proteste unterstützten (Tsikata/Yaro 2011: 21). Die Landnutzer*innen in den Dörfern, unabhängig davon ob sie Indigene oder Migrant*innen waren, tauschten sich untereinander über die Missstände aus, überließen weitere Schritte jedoch den jeweiligen sub-chiefs. Die Landnutzer*innen und sub-chiefs der settler communities waren sich bewusst, dass

63 Landnutzer*innen aus Jimle und Gumani, Gruppendiskussion am 10.5.2016; Landnutzer*innen und settler-chief aus Kpachaa, Gruppendiskussion am 11.5.2016; Landnutzer und settler-chief aus Parachanayilli, Gruppendiskussion am 13.5.2016; Landnutzer*innen und sub-chief von Tuya, Gruppendiskussion am 14.5.2016; Landnutzer*innen und sub-chief von Jashei, Gruppendiskussion am 15.5.2016. 
sie nichts gegen die Nutzung des Lands durch das Unternehmen tun konnten. Aussagen wie »Es hat uns geärgert, dass das Unternehmen unser Land genommen hat, aber wir konnten nichts dagegen sagen ${ }^{64}$ oder »Wir haben nicht gekämpft, da wir settler farmer sind und das Land uns nicht gehört « ${ }^{65}$ verdeutlichen das. Die anderen sub-chiefs wandten sich mit ihren Beschwerden an den Tijo Naa. Sie machten auf ihren Unmut und die wahrgenommenen Missstände aufmerksam und bekamen Verständnis entgegengebracht. An der Situation änderte sich dadurch jedoch nichts.

64 Landnutzer und settler-chief aus Parachanayilli, Gruppendiskussion am 13.5.2016.

65 Landnutzer*innen und settler-chief aus Kpachaa, Gruppendiskussion am 11.5.2016. 
